\title{
Hawking radiation as quantum tunneling
}

\section{K. Saifullah*}

Department of Mathematics, Quaid-i-Azam Univerity, Islamabad, Pakistan

E-mail: saifullah@qau.edu.pk

\section{Jamil Ahmed}

Department of Mathematics, Quaid-i-Azam Univerity, Islamabad, Pakistan

E-mail: jahmedestudent.qau.edu.pk

Tunneling method as a semi-classical way is discussed, that gave insight into Hawking radiations. The approach which we have used to study is robust. It is applicable for a variety of spherically and cylindrically symmetric solutions of Einstein's field equations to calculate the temperature of Hawking radiations consisting of scalar and fermionic particles. In this approach we have used Hamilton Jacobi method for the calculation of classical action and tunneling probability for black strings and black holes. Finally, greybody factor as a characteristic of Hawking radiation will also be discussed.

36th International Cosmic Ray Conference -ICRC2019-

July 24th - August 1st, 2019

Madison, WI, U.S.A.

${ }^{*}$ Speaker. 


\section{Introduction}

Classically black holes are considered as such dense objects in nature that nothing could escape out from them. The major development in this area of black hole physics occurred when Stephen Hawking pointed out that quantum mechanically black hole could radiate [1, 2]. He argued that due to strong gravitational field and vacuum fluctuations at the event horizon of the black hole, a particle anti-particle pair is created. One will go inside and other will tunnel out and this will appear to an outside observer that black hole have emitted a particle. The idea given by Hawking was based on quantum field theory in curved spacetimes. This idea give rise to a whole new field in theoretical physics. As yet there is no full quantum description of black holes developed, so people have tried some semi-classical approaches to study these radiations. Initially this approach was developed for Schwarzchild black hole $[3,4,5]$. then it was extended successfully for a wide variety of spherically symmetric black holes $[6,7,8,9,10,11,12,13,14,15]$. this extensive litrature showed that this approach is quite robust mathematically. In this technique, we calculate the imaginary part of the classical action that give the rate of emission and absorption of particles across a black hole $[23,6]$. Using this approach we can calculate the temperature of Hawking radiations.

The framework available in litrature mentioned above and some of our's work in this direction was to study was to study Hawking radiations from spherically symmetric configurations. We have also applied this approach to cylindrically symmetric spacetimes as well. It is worthless to mention here that in case of charged rotating black strings, using this technique we have corrected the form of temperature that was available for charged black strings.

The spectrum of Hawking radiations from black holes and black strings comprises of different particles that includes scalar particles. fermions, gravitons etc. We have done this work as general frame work that is applicable to study raditions comprises of all such particles one by one. In [17] we have studied fermionic tunneling from black strings, in [18] we have extended this to study Hawking radiations of fermions froms charged rotating black strings. Further in [19], we have studied Hawking radiations of scalar particles from dilatonic black holes. In [20] we have studied Hawking radiations of scalar particles from black strings. In [21] tunneling of gravitons is discussed.

\section{Tunneling approach}

The technique was intended to be a straightforward semi-classical model that would give intuitive insight into Hawking radiation. Since the model is only semi-classical, it is not immediately apparent that it would be particularly robust when applied to a large variety of spacetimes [6]. In order to demonstrate the effectiveness of tunneling method Kerner and Mann applied this method to a variety of different spherically symmetric spacetimes. Lemos was the first who showed that cylindrically symmetric rotating black hole solution of Einsteins field equations with a negative cosmological constant do indeed exist [22]. These cylindrical black holes are also called black strings. Hawking discovered the thermal radiation of a collapsing black hole using the techniques of quantum field theory in curved spacetime [5]. In recent years, a semi-classical derivation of the Hawking radiation as a tunneling process has been developed by Wilczek and Parikh [3]. Zhang 
and Zhao extended this method to the Reissner- Nordström black hole [13] and Kerr-Newman black holes [8]. Angheben et al also proposed a derivation of the Hawking radiation by calculating the particles classical action from the Hamilton-Jacobi equations, which is an extension of the complex path analysis of Padmanabhan et al [16]. All these approaches to the tunneling method used the fact that the tunneling probability of the classically forbidden trajectory from inside to outside the horizon is given by

$$
\Gamma=\exp \left(\frac{-2}{\hbar} \operatorname{ImI}\right),
$$

where $I$ is classical action of the trajectory. There are two methods available to calculate the classical action, the Hamilton Jacobi method and null geodesic method. A comparison of these two methods is given by Kerner and Mann [6]. We will use the Hamilton Jacobi method to calculate classical action of black string and their temperature at horizon. To illustrate this we present a working procedure, how to calaculate classical action and Hawking temperature.

\section{Illustration of tunneling method}

We describe the method for the classical action for fermionic tunneling from any black hole. For which we have to consider Dirac equation given by,

$$
i \gamma^{\mu}\left(D_{\mu}-\frac{\imath q}{\hbar} A_{\mu}\right) \Psi+\frac{m}{\hbar} \Psi=0,
$$

where $m, q$ and $A_{\mu}$ are the mass, charge and electromagnetic potential of the particle, and

$$
D_{\mu}=\partial_{\mu}+\Omega_{\mu}=\partial_{\mu}+\frac{1}{2} l \Gamma_{\mu}^{\alpha \beta} \Sigma_{\alpha \beta}
$$

Following the definitions $\Sigma_{\alpha \beta}=-\Sigma_{\alpha \beta}$; when $\alpha \neq \beta$ and $\Sigma_{\alpha \alpha}=0 ; \alpha=\beta$ we have

$$
\Omega_{\mu}=\frac{1}{2} l \Gamma_{\mu}^{\alpha \beta} \Sigma_{\alpha \beta}=0
$$

So

$$
D_{\mu}=\partial_{\mu}
$$

The gamma matrices are defined from the background black hole such that they obey the following properties,

$$
\left\{\gamma^{\mu}, \gamma^{v}\right\}=2 g^{\mu v}
$$

where $g^{\mu v}$ are is inverse of metric tensor. Consider the ansatz [14, 7]

$$
\begin{gathered}
\Psi_{\uparrow}(t, r, \theta, \phi)=\left(\begin{array}{c}
A(t, r, \theta, \phi) \xi_{\uparrow} \\
B(t, r, \theta, \phi) \xi_{\uparrow}
\end{array}\right) \exp \left[\frac{l}{\hbar} I_{\uparrow}(t, r, \theta, \phi)\right], \\
\Psi_{\uparrow}(t, r, \theta, z)=[] A(t, r, \theta, z) \xi_{\uparrow} B(t, r, \theta, z) \xi_{\uparrow} \exp \left(\frac{l}{\hbar} I(t, r, \theta, z)\right),
\end{gathered}
$$

where $\xi_{\uparrow}=\left(\begin{array}{l}1 \\ 0\end{array}\right)$ and $I(t, r, \theta, z)$ is part of classical action. It can be split considering the symmetries of background spacetimes symmetries. For cylindrically symmetric spacetimes it can be wriitten as, 


$$
I_{\uparrow}(t, r, \theta, z)=-E t+l \theta+J z+W(r),
$$

where $E$ is the energy of the emitted particles and $W$ is the part of the action $I_{\uparrow}$ that contributes to the tunneling probability. The probabilities of crossing the horizon in each direction are proportional to [7]

$$
\begin{aligned}
& \operatorname{Prob}(\text { out })=\exp (-2 \operatorname{ImI})=\exp \left[-2\left(\operatorname{Im} W_{+}+\operatorname{Im} \Phi\right)\right] \\
& \operatorname{Prob}(\text { in })=\exp (-2 \operatorname{ImI})=\exp \left[-2\left(\operatorname{Im} W_{-}+\operatorname{Im} \Phi\right)\right] .
\end{aligned}
$$

While computing the imaginary part of the action, we note that it is same for both the incoming and outgoing solutions, and so will cancel out in computing the emission probability. Now the probability of particles tunneling from inside to outside the horizon is given by [7]

$$
\Gamma \propto \frac{\operatorname{Prob}(\text { out })}{\operatorname{Prob}(\text { in })}=\frac{\exp \left[-2\left(\operatorname{Im} W_{+}+\operatorname{Im} \Phi\right)\right]}{\exp \left[-2\left(\operatorname{Im} W_{-}+\operatorname{Im} \Phi\right)\right]},
$$

or

$$
\Gamma=\frac{\exp \left(-2 \operatorname{Im} W_{+}\right)}{\exp \left(-2 \operatorname{Im} W_{-}\right)}=\exp \left(-4 \operatorname{Im} W_{+}\right)
$$

Expanding the action in terms of particle's energy [7] we get

$$
\Gamma=\exp (-\beta E)
$$

where

$$
\beta=\frac{1}{T_{H}},
$$

from where we get analytical expression for Hawking temperature of the radiations emitted from background black hole.

\section{Greybody factor}

If one considers black holes as a thermal system, then black holes will have temperature and entropy. This implies that black holes can radiate. As thermal systems, black holes have an associated temperature and entropy and therefore they radiate, and the radiations are called Hawking radiations. The emission rate in a mode of frequency $\omega$, at the event horizon, is given by

$$
\Gamma(\omega)=\left(\frac{1 d^{3} k}{e^{\beta \omega} \pm 1(2 \pi)^{3}}\right) .
$$

In this relation $\beta$ is used to denote the inverse of the Hawking temperature and minus (plus) sign is for bosons (fermions, respectively). This formula for emission rate can be generalized for any dimension and it is valid for massive and massless particles. Spectrum of the radiations from black holes at the event horizon is perfectly same as that of the black body spectrum. Due to this, it gives rise to the information loss paradox. The important fact is that the geometry of the spacetime around a black is non-trivial. This non-trivial geometry modifies the spectrum of Hawking radiations. In fact, the non-trivial geometry acts as a potential barrier which allows some of the radiations to 
transmit and reflect the rest to the black hole. The mathematical expression that summarizes all the above discussion is

$$
\Gamma(\omega)=\left(\frac{\gamma(\omega) d^{3} k}{e^{\beta \omega} \pm 1(2 \pi)^{3}}\right),
$$

where $\gamma(\omega)$ is known as the greybody factor, which is frequency-dependent.

Physically, greybody factor originates from an effective potential barrier by a black hole spacetime. For example, the potential barrier for massless scalars from Schwarzschild spacetime is

$$
V_{e f f}(r)=\left(1-\frac{r_{H}}{r}\right)\left(\frac{r_{H}}{r^{3}}+\frac{l(l+1)}{r^{2}}\right),
$$

where $r_{H}$ is the horizon's radius and $l$ is angular momentum of the scalar. It is this potential which transmits or reflects radiations from black holes. Therefore, it gives rise to the frequency dependent greybody factor.

Greybody factor not only accounts for the deviation of Hawking radiations from black body spectrum, but is also important in working out energy emission rates and is also relevant for computing the partial absorption cross sections of black holes.

\section{Conclusions}

Hawking radiations can be viewed as a quantum tunneling process and should contain contributions of both scalar particles and fermions. Kerner and Mann have presented fermion's radiation for Rindler spacetime and for other spherically symmetric black holes. In order to extend our understanding on Hawking radiations, we have discussed it as a general framework to study radiations emitted from spherically as well as cylindrically symmetric black holes. An extensive literature in this regard is cited, which shows the universality and effectiveness of this technique. This is more convenient way to analyse the Hawking radiations. Spectrum of Hawking radiations differs from that of blackbody radiations due to a frequency dependent factor known as greybody factor. Physical origin of greybody factor is also discussed in this paper.

\section{References}

[1] S. W. Hawking, Commun. Math. Phys. 43 (1975) 199.

[2] S. W. Hawking, Nature 248 (1974) 30.

[3] M. K. Parikh and F. Wilczek, Phys. Rev. Lett. 85 (2000) 5042.

[4] P. Kraus and F. Wilczek, Nucl. Phys. B 433 (1995) 403.

[5] M. K. Parikh, Gen. Rel. Grav. 36 (2004) 2419.

[6] R. Kerner and R. B. Mann, Phys. Rev. D 73 (2006) 104010.

[7] R. Kerner and R. B. Mann, Class. Quant. Grav. 25 (2008) 095014

[8] S. Zhou and W. Liu, Phys. Rev. D 77 (2008) 104021.

[9] R. Li, J. R. Ren and S. W. Wei, Class. Quant. Grav. 25 (2008) 125016.

[10] D. Y. Chen, Q. Q. Jiang and X. T. Zu, Class. Quant. Grav. 25 (2008) 205022. 
[11] Q. Q. Jiang, Phys. Lett. B 666 (2008) 517.

[12] C. Ding and J. Jing, Class. Quant. Grav. 27 (2010) 035004.

[13] J. Yang and S. Z. Yang, J. Geom. Phys. 60 (2010) 986.

[14] U. A. Gillani and K. Saifullah, Phys. Lett. B 699 (2011) 15.

[15] M. Rehman and K. Saifullah, JCAP 03 (2011) 001.

[16] K. Srinivasan and T. Padmanabhan, Phys. Rev. D 60 (1999) 24007.

[17] J. Ahmad and K. Saifullah, JCAP 08 (2011) 011.

[18] J. Ahmad and K. Saifullah, JCAP 011 (2011) 023.

[19] H. Gohar and K. Saifullah, Gen. Relativ. Gravit. 44 (2012) 3163.

[20] H. Gohar and K. Saifullah, Astrophys. Space Sci. 12 (2012) 1255.

[21] H. Lin, K. Saifullah and S-T. Yau, Mod. Phys. Lett. A 30 (2015) 1550044.

[22] J. P. S. Lemos, Phys. Lett. B 353 (1995) 46.

[23] S. Shankaranarayanan, T. Padmanabhan and K. Srinivasan, Class. Quant. Grav. 19 (2002) 2671. 